OPEN ACCESS

Edited by:

Brian Ahmer,

The Ohio State University,

United States

Reviewed by:

Joshua D. Shrout,

University of Notre Dame,

United States

Michelle Visser,

University at Buffalo, United States

*Correspondence:

Hengmi Cui

hmcui@yzu.edu.cn

Guoqiang Zhu

yzgqzhu@yzu.edu.cn

Received: 29 January 2018 Accepted: 24 April 2018 Published: 09 May 2018

Citation:

Yang Y, Zhou M, Hardwidge PR, Cui H and Zhu $G$ (2018) Isolation and

Characterization of $\mathrm{N}$-acyl Homoserine Lactone-Producing Bacteria From Cattle Rumen and Swine Intestines. Front. Cell. Infect. Microbiol. 8:155. doi: 10.3389/fcimb.2018.00155

\section{Isolation and Characterization of N-acyl Homoserine Lactone-Producing Bacteria From Cattle Rumen and Swine Intestines}

\author{
Yang Yang ${ }^{1,2,3}$, Mingxu Zhou ${ }^{2,3}$, Philip R. Hardwidge ${ }^{4}$, Hengmi Cui ${ }^{\text {** }}$ and Guoqiang Zhu ${ }^{2,3 *}$ \\ ${ }^{1}$ Institute of Epigenetics and Epigenomics and College of Animal Science and Technology, Yangzhou University, Yangzhou, \\ China, ${ }^{2}$ Jiangsu Co-Innovation Center for Important Animal Infectious Diseases and Zoonoses, Yangzhou University, \\ Yangzhou, China, ${ }^{3}$ College of Veterinary Medicine, Yangzhou University, Yangzhou, China, ${ }^{4}$ College of Veterinary Medicine, \\ Kansas State University, Manhattan, KS, United States
}

Quorum sensing systems regulate gene expression in response to bacterial population density. Acyl-homoserine lactones are a class of quorum sensing molecules found in cattle rumen that are thought to regulate the gene expression of enterohemorrhagic Escherichia coli and thus help this pathogen survive in animal gastrointestinal tracts. However, the specific bacteria that produce these signaling molecules in bovine and porcine gastrointestinal tracts are unknown. Here we developed methods to concentrate gastrointestinal fluids and screen the bacteria that produce acyl-homoserine lactones. We isolated a Pseudomonas aeruginosa strain YZ1 from cattle rumen, and an Aeromonas hydrophila strain YZ2 from pig intestine. Mass spectrometry analysis of culture supernatants indicated at least three specific classes of acyl-homoserine lactones produced by $\mathrm{YZ1}$, and a C4-acyl-homoserine lactone produced by $\mathrm{YZ2}$. Transformation of $E$. coli with $P$. aeruginosa or $A$. hydrophila luxl homologs, which can produce short- or long-chain acyl-homoserine lactones conferred upon $E$. coli the ability to synthesize acyl-homoserine lactones and affected gene expression, motility, and acid tolerance of $E$. coli. This is the first study reporting the isolation and characterization of acyl-homoserine lactone synthase-positive bacteria from cattle rumen and swine intestines.

Keywords: acyl-homoserine lactone, cattle rumen, E. coli, pig intestine, quorum sensing

\section{INTRODUCTION}

Quorum sensing (QS) systems regulate gene expression in response to bacterial population density. Small molecules named autoinducers (AIs) are produced, released, and detected in the QS process. The LuxI/LuxR QS-I system was first described in the bioluminescent marine bacterium Vibrio fischeri. When QS-I threshold concentrations are reached, diverse biological processes are affected, including symbiosis, virulence, competence, conjugation, antibiotic production, motility, sporulation, and biofilm formation (Reading and Sperandio, 2006; Walters and Sperandio, 2006; Waters et al., 2008; Boyen et al., 2009).

Cattle are the primary animal reservoir of enterohemorrhagic E. coli (EHEC) (Andrade et al., 2012; Borriello et al., 2012; Duan et al., 2012, 2013; Yang et al., 2013), an important foodborne 
TABLE 1 | Strains and plasmids used in this study.

\begin{tabular}{|c|c|c|}
\hline Strain/plasmid & Description & Source/references \\
\hline \multicolumn{3}{|l|}{ STRAINS } \\
\hline A. tumefaciens JZA1 & AHL biosensor & $\begin{array}{l}\text { Fuqua and Winans, } \\
1996\end{array}$ \\
\hline C. violaceum CV026 & AHL biosensor & Latifi et al., 1995 \\
\hline Escherichia coli pSB401 & AHL biosensor & Winson et al., 1998 \\
\hline E. coli pSB1142 & AHL biosensor & Winson et al., 1998 \\
\hline E. coli O157:H7 8624 & Wild-type EHEC & Havens et al., 1992 \\
\hline E. coli $107 / 86$ & Wild-type STEC & $\begin{array}{l}\text { Bertschinger et al., } \\
1990\end{array}$ \\
\hline E. coli 8624/pBR322 & $\begin{array}{l}\text { E. coli } 8624 \\
\text { carrying pBR322 }\end{array}$ & This study \\
\hline E. coli 8624/p27853-las/ & $\begin{array}{l}\text { E. coli } 8624 \\
\text { carrying } \\
\text { p27853-las/ }\end{array}$ & This study \\
\hline E. coli 8624/p27853-rhll & $\begin{array}{l}\text { E. coli } 8624 \\
\text { carrying } \\
\text { p27853-rhll }\end{array}$ & This study \\
\hline E. coli 8624/pYZ1-las/ & $\begin{array}{l}\text { E. coli } 8624 \\
\text { carrying pYZ1-las/ }\end{array}$ & This study \\
\hline E. coli 8624/pYZ1-rhll & $\begin{array}{l}\text { E. coli } 8624 \\
\text { carrying pYZ1-rhll }\end{array}$ & This study \\
\hline E. coli 107/86/pBR322 & $\begin{array}{l}\text { E. coli } 107 / 86 \\
\text { carrying pBR322 }\end{array}$ & This study \\
\hline E. coli 107/86/pJ1-ahyl & $\begin{array}{l}\text { E. coli 107/86 } \\
\text { carrying pJ1-ahyl }\end{array}$ & This study \\
\hline E. coli 107/86/pYZ2-ahyl & $\begin{array}{l}\text { E. coli 107/86 } \\
\text { carrying pYZ2-ahyl }\end{array}$ & This study \\
\hline $\begin{array}{l}\text { Pseudomonas aeruginosa } \\
\text { ATCC27853 }\end{array}$ & lasl/rhll cloning & D'Amato et al., 1975 \\
\hline$P$. aeruginosa $Y Z 1$ & $\begin{array}{l}\text { Strain isolated } \\
\text { from cattle rumen }\end{array}$ & This study \\
\hline A. hydrophila J-1 & ahyl cloning & Yan et al., 1996 \\
\hline A. hydrophila YZ2 & $\begin{array}{l}\text { Strain isolated } \\
\text { from pig intestines }\end{array}$ & This study \\
\hline $\begin{array}{l}\text { Yersinia enterocolitica } \\
\text { GIM1.266 }\end{array}$ & $\begin{array}{l}\text { Al-1 bioassay } \\
\text { positive control }\end{array}$ & $\begin{array}{l}\text { Guangdong Inst. } \\
\text { Microbiology }\end{array}$ \\
\hline \multicolumn{3}{|l|}{ PLASMIDS } \\
\hline pBR322 & $\begin{array}{l}\text { Expression vector, } \\
\text { Amp }^{r}\end{array}$ & Takara Ltd. \\
\hline pJ1-ahyl & $\begin{array}{l}\mathrm{J}-1 \text { ahyl in } \\
\text { pBR322 }\end{array}$ & This study \\
\hline pYZ2-ahyl & $\begin{array}{l}\text { YZ2 ahyl in } \\
\text { pBR322 }\end{array}$ & This study \\
\hline p27853-las/ & $\begin{array}{l}\text { ATCC27853 las/ in } \\
\text { pBR322 }\end{array}$ & This study \\
\hline p27853-rhll & $\begin{array}{l}\text { ATCC27853 rhll in } \\
\text { pBR322 }\end{array}$ & This study \\
\hline pYZ1-lasl & $\begin{array}{l}\text { YZ1 lasl in } \\
\text { pBR322 }\end{array}$ & This study \\
\hline pYZ1-rhll & YZ1 rhll in pBR322 & This study \\
\hline
\end{tabular}

pathogen that causes outbreaks of hemorrhagic colitis and hemolytic uremic syndrome (Barlow and Mellor, 2010; Sperandio, 2010). Shiga-like toxin producing (SLTEC) and verotoxigenic E. coli (VTEC) cause both porcine edema disease (ED) and post-weaning diarrhea (PWD) (da Silva et al., 2001; Frydendahl, 2002; Mainil et al., 2002). E. coli encodes a single
TABLE 2 | Primers used in this study.

\begin{tabular}{|c|c|}
\hline Primer & Sequence $\left(5^{\prime}-3^{\prime}\right)$ \\
\hline $16 \mathrm{SF}$ & AGAGTTGATCCTGGCTCAG \\
\hline $16 S R$ & GGCTACCTTGTTACGACT \\
\hline rhll-F & CGCGGATCCATGATCGAATTGCT \\
\hline rhlll-R & TTAGTCGACTCACACCGCCATCG \\
\hline lasl-F & CGCGGATCCATGATCGTACAAATT \\
\hline lasl-R & TTAGTCGACTCATGAAACCGCCAGT \\
\hline ahyl-F & CGAGCTAGCATGCTTGTITCAAAG \\
\hline ahyl-R & TAAGTCGACTTATTCGGTGACCAGT \\
\hline fliC-EHEC-RT-F & TGTGACTGTTGCCGGGTATG \\
\hline fliC-EHEC-RT-R & AGTGATITACCCGCGGAGTT \\
\hline fliC-STEC-RT-F & CAGCAAGCGGTGAAGTGAA \\
\hline fliC-STEC-RT-R & AAGCGTAGCCACAGTAGCA \\
\hline sdiA-RT-F & GCGTCGCACGATGCTGTT \\
\hline sdiA-RT-R & CCCACGCCTCAGGGTAAT \\
\hline gadA-RT-F & AGGCAAACCAACGGATAAACC \\
\hline gadA-RT-R & GGCGCATAGGGATCTCACG \\
\hline gapA-RT-F & CGTTAAAGGCGCTAACTTCG \\
\hline gapA-RT-R & ACGGTGGTCATCAGACCTTC \\
\hline
\end{tabular}

LuxR homolog named SdiA, but is believed not to express the LuxI homolog, the acyl-homoserine lactone (AHL) synthase, which produces AI-1 (Ahmer, 2004; Yao et al., 2006; Smith et al., 2011). In the presence of exogenous AHLs, a significant proportion of SdiA, the AHL receptor, is expressed in a folded, soluble form, while it is expressed in insoluble inclusion bodies in the absence of AI-1. Thus, E. coli can regulate its virulence gene expression in response to the population density of other AHL-positive bacteria (Dyszel et al., 2010; Soares and Ahmer, 2011; Yakhnin et al., 2011). These responses include cell division (Yamamoto et al., 2001; Shimada et al., 2014), antibiotic resistance (on plasmid-encoded sdiA condition) (Rahmati et al., 2002; Van Houdt et al., 2006; Dyszel et al., 2010; Lu et al., 2017; Zhang et al., 2018), motility (Kanamaru et al., 2000; Wei et al., 2001; Yang et al., 2013), and the activity of other QS systems (Yang et al., 2013).

Different AHLs (C6-HSL, C8-HSL) have been detected in cattle rumens (Erickson et al., 2002; Hughes et al., 2010). QS signals in the rumen repress the expression of the locus of enterocyte effacement $(L E E)$ genes and activate the expression of the glutamate decarboxylase A ( $g a d A)$ gene in EHEC, suggesting QS-I signals modulate EHEC passage through the bovine gastrointestinal tract (Dyszel et al., 2010; Sperandio, 2010; Sheng et al., 2013; Nguyen et al., 2015), perhaps influencing EHEC survival in acidic environments. However, the specific source of these QS-1 signals is currently lacking, as, to our knowledge, there are no reports of the isolation and identification of AHL-producing bacteria from cattle rumen (Erickson et al., 2002; Soares and Ahmer, 2011).

AHL activities in vivo have been examined previously from the gastrointestinal tracts of various animals, including pigs, using a reporter strain method (Smith et al., 2008). The lack of evidence for AHL-producing bacteria in pig intestines has 


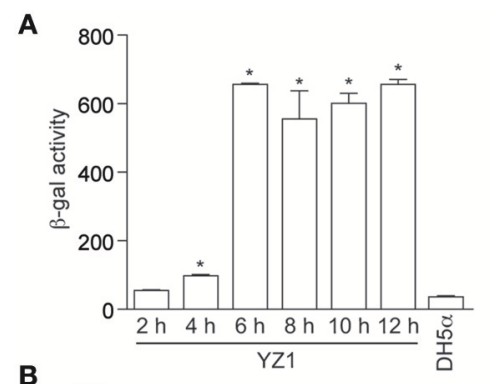

B

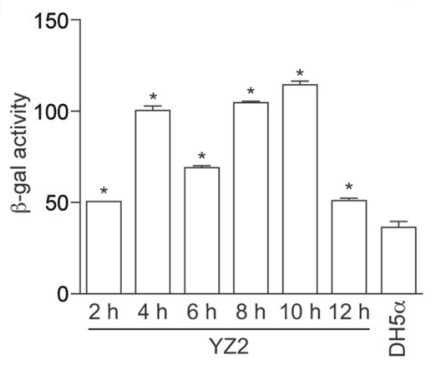

C

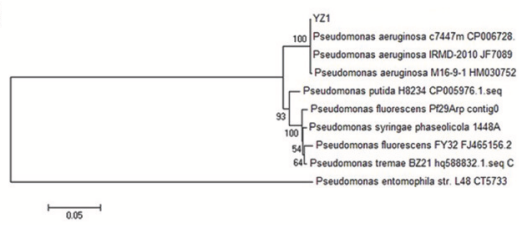

D

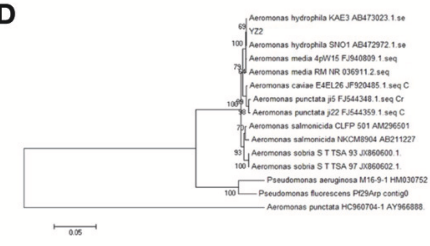

E

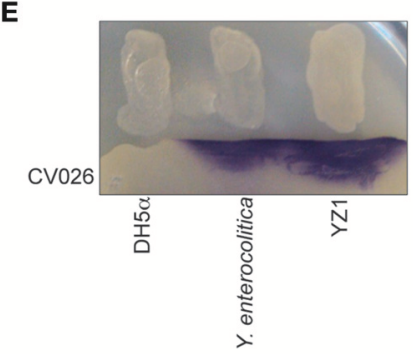

$\mathbf{F}$

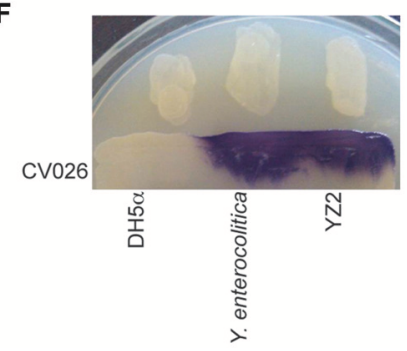

FIGURE 1 | Isolation of the AHL-producing bacteria $Y Z 1$ and $Y Z 2$. (A,B) JZA1-based $\beta$-galactosidase assays of $A H L$ production. $\beta$-galactosidase activity is plotted as a function of the culture supernatant added to JZA1 cultures. Strains (YZ1, A; YZ2, B) were grown for different times before the addition of supernatants, as indicated. Asterisks indicate significantly different
FIGURE 1 | $\beta$-galactosidase activity as compared with activity induced by DH5 $\alpha$ supernatant. (C,D) Phylogenetic analyses of YZ1 and YZ2. The bar represents evolutionary distance as number of changes per nucleotide position, determined by measuring the lengths of the horizontal lines connecting the corresponding species. GenBank accession numbers are indicated. (E,F) Biosensor strain C. violaceum CV026-based cross-streaking assays for Al-1 activity (purple color). P. aeruginosa strains YZ1 (E) A. hydrophila YZ2 (F) the positive control Y. enterocolitica GIM1.266, and the negative control $\mathrm{DH} 5 \alpha$ were struck across the biosensor strain as indicated.

also hindered in-depth studies of QS-I function on E. coli in pigs. The QS-II system has been studied in porcine (Zhu et al., 2011; Yang et al., 2014), bovine (Sperandio et al., 2002), and avian (Palaniyandi et al., 2013) pathogenic E. coli, and has been shown to affect virulence gene expression. Here we developed a method with which to concentrate gastrointestinal tract samples and screen the contents for AHL-producing bacteria. We isolated and characterized an $\mathrm{N}$-acyl homoserine lactone-producing Pseudomonas aeruginosa strain from cattle and an Aeromonas hydrophila strain from pigs. We also determined the extent to which QS-I signals from these strains impact E. coli virulence gene expression in vitro.

\section{MATERIALS AND METHODS}

\section{Bacterial Strains and Growth Conditions}

The bacterial strains and plasmids used are listed in Table $\mathbf{1 .}$ E. coli 107/86 (wild-type, O139:H1: F18ab, Stx2e) (Bertschinger et al., 1990) was cultured in Luria broth (LB) or on Luria agar (LA) plates at $37^{\circ} \mathrm{C}$. A. hydrophila J-1 (Yan et al., 1996) was used as positive control for ahyI gene expression. Yersinia enterocolitica GIM1.266 was used as a positive control in AHL cross-streaking assays. E. coli $\mathrm{DH} 5 \alpha$ was used as a negative control. Agrobacterium tumefaciens JZA1 (Fuqua and Winans, 1996) and Chromobacterium violaceum CV026 (Latifi et al., 1995) were cultured in Luria broth (LB) or on Luria agar (LA) plates at $30^{\circ} \mathrm{C}$. The bioluminescence reporter strains E. coli pSB401 and pSB1142 (Winson et al., 1998) were used as short- and longside chain AHLs biosensors, respectively, and were grown in $\mathrm{LB}$ at $37^{\circ} \mathrm{C}$.

\section{Isolation of AHL-Producing Bacteria}

Cattle rumen fluids from cattle rumen fistula and pig intestinal samples were obtained from the Animal Hospital of Yangzhou University according to the Guide for the Care and Use of Laboratory Animals of the Ministry of Science and Technology of the People's Republic of China. The protocols for animal experiments were approved by the Jiangsu Administrative Committee for Laboratory Animals (approval number: SYXKSU-2007-0005), and complied with the guidelines of Jiangsu laboratory animal welfare and ethics of Jiangsu Administrative Committee of Laboratory Animals. Rumen contents were filtered through six layers of hospital gauze. The filtered rumen fluid and PBS-washed intestinal samples were centrifuged at $10,000 * \mathrm{~g}$ for $10 \mathrm{~min}$, and the pellets were spread on LB plates and incubated at $37^{\circ} \mathrm{C}$ for $24 \mathrm{~h}$. Isolated bacterial colonies were inoculated 

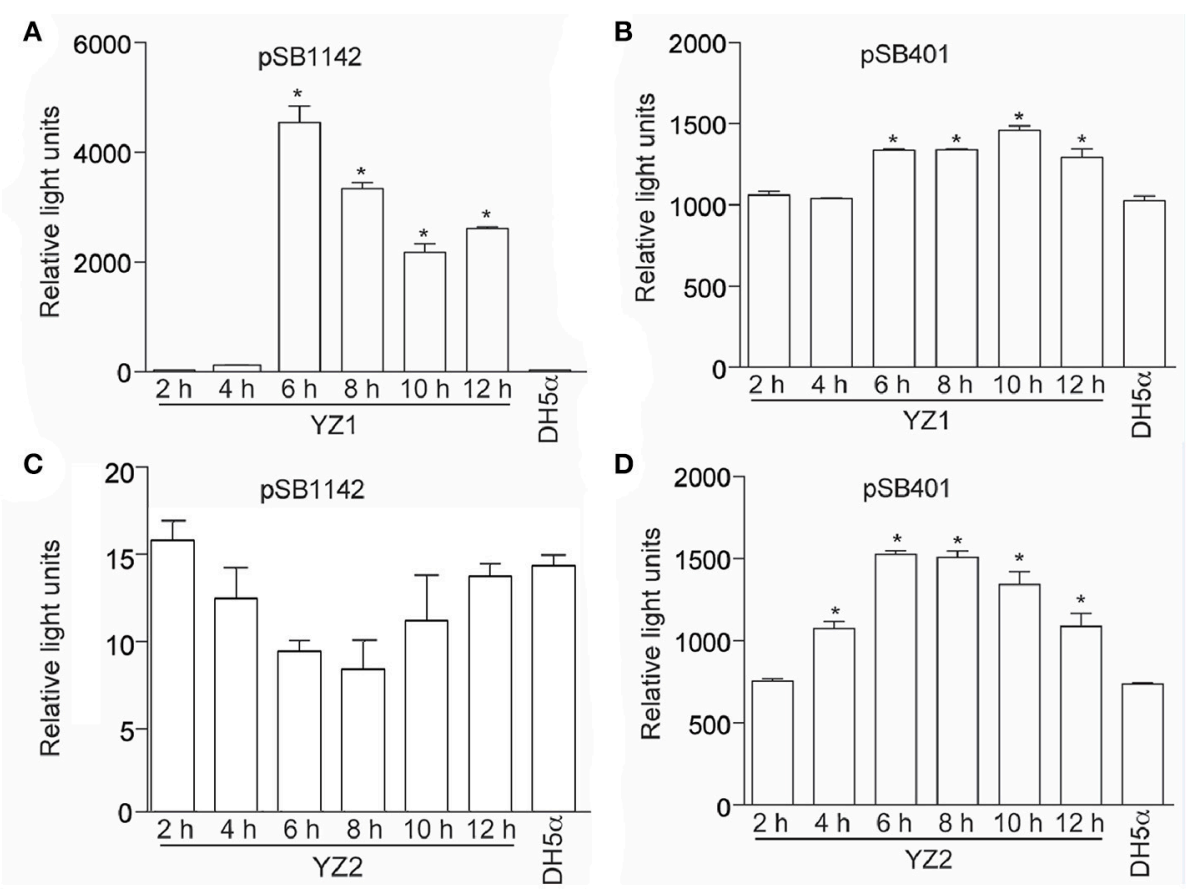

FIGURE 2 | Bioluminescence assays of AHL production. (A) Bioluminescence (relative light units) of reporter strain E. coli pSB1142 after the addition of the indicated culture supernatants from YZ1. (B) Bioluminescence (relative light units) of reporter strain E. coli pSB401 after the addition of the indicated culture supernatants from YZ1. (C) Bioluminescence (relative light units) of reporter strain E. coli pSB1142 after the addition of the indicated culture supernatants from YZ2. (D) Bioluminescence (relative light units) of reporter strain E. coli pSB401 after the addition of the indicated culture supernatants from YZ2. Asterisks indicate significantly different reporter strain bioluminescence as compared with activity induced by $\mathrm{DH} 5 \alpha$ supernatant.

into $8 \mathrm{ml} \mathrm{LB}$ for overnight growth, after which the culture supernatants were extracted in $2 \mathrm{ml}$ ethyl acetate (Sperandio, 2010). The organic phase was collected and evaporated to dryness. Twenty microliters of Milli-Q water was added to dissolve the dried contents, then seeded onto a $0.8 \%$ LA plates spread with both $\mathrm{X}$-gal $(50 \mu \mathrm{g} / \mathrm{ml})$ and the reporter strain JZA1 $\left(5 \times 10^{7} \mathrm{CFU} / \mathrm{ml}\right)$.

\section{Cross-Streaking Assay}

Cross-streaking assays were performed as described previously (Yang et al., 2013). The reporter strain CV026 was spread in the middle of an LA plate, and tested strains were streaked perpendicular to CV026. After overnight growth, purple color induced in CV026 was observed visually. Similarly, a JZAI-based crossstreaking assay for long-side chain AHLs detection was conducted.

\section{Strain Phylogenetic Analysis}

The partial 16S rDNA genes from YZ1 and YZ2 were amplified using PCR (Chen et al., 2013) with primers 16SF and 16SR (Table 2) and subsequently sequenced. Related nucleotide sequences available in GenBank were aligned and phylogenetic analysis was conducted using MEGA 4.0.

\section{AHL Extraction}

To obtain sufficient AHLs for biochemical characterization, YZ1 and YZ2 were inoculated into $50 \mathrm{ml} \mathrm{LB}$ respectively and, from 2 to $12 \mathrm{~h}$ growth, were extracted vigorously with ethyl acetate as described (Chen et al., 2013). The organic phases were collected and filtered through a $0.22 \mu \mathrm{m}$ filter membrane, evaporated, and dissolved in $100 \mu \mathrm{l}$ of Milli-Q water.

\section{$\beta$-Galactosidase Assays}

An overnight culture of JZA1 $\left(2 \times 10^{9} \mathrm{CFU} / \mathrm{ml}\right)$ was diluted 1:100 into $1 \mathrm{ml}$ fresh LB medium and $100 \mu \mathrm{l}$ extracts of bacterial cultures or rumen fluids were added. After $12 \mathrm{~h}$ incubation, $\beta$-galactosidase activity for each sample was determined using ONPG as described (Tran et al., 2001).

\section{Bioluminescence Assays}

The $l u x$-based biosensor E. coli strains pSB401 and pSB1142 were used to determine inducible lux bioluminescence activity activated by short- and long-side chain AHLs, respectively (Winson et al., 1998). Overnight cultures of E. coli pSB401 and pSB1142 were diluted 1:100 into $1 \mathrm{ml}$ fresh LB medium and 100 $\mu \mathrm{l}$ extracts of bacterial cultures or rumen fluids were added. After $6 \mathrm{~h}$ incubation, bioluminescence was measured using a Tecan GENios Plus microplate reader in luminescence mode (TECAN $\mathrm{GmbH}$, Austria). Data were expressed as relative light units of luminescence values from each sample. 


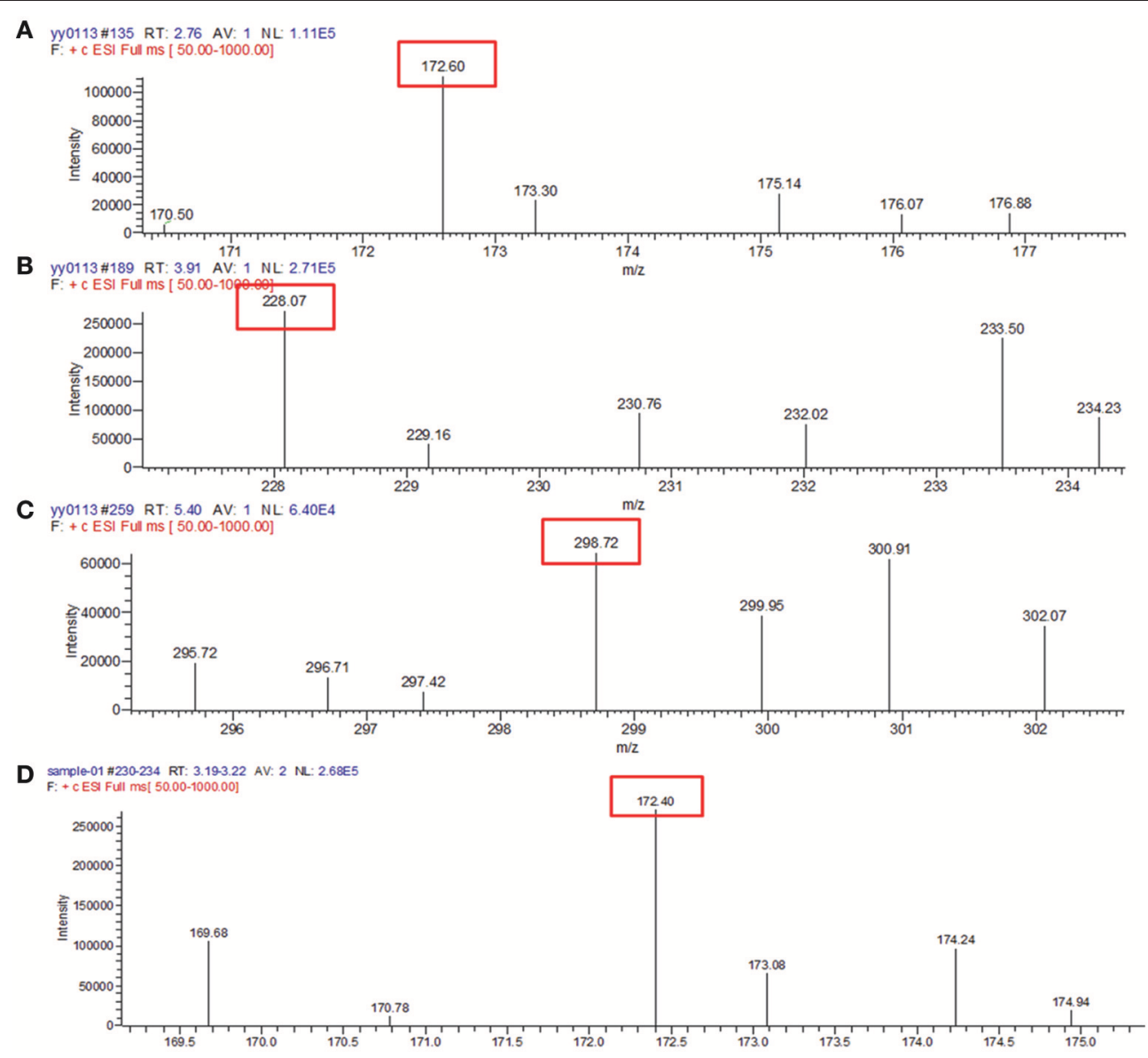

FIGURE 3 | Mass spectrometry analysis of AHLs in YZ1 and YZ2 extracts. (A) Identification of C4-HSL produced by YZ1. (B) Identification of C8-HSL produced by YZ1. (C) Identification of 3-oxo-C12-HSL produced by YZ1. (D) Identification of C4-HSL produced by YZ2.

\section{Mass Spectrometry}

High performance liquid chromatography mass spectrometry was used to analyze AHL production by YZ1 and YZ2 using a LCQ Deca XP max (Thermo Finnigan, USA; Yin et al., 2012; Chen et al., 2013). Liquid chromatography steps utilized a C18 column of $4.6 \times 150 \mathrm{~mm}, 5 \mu \mathrm{m}$ particle size, with injection volume of $20 \mu \mathrm{l}$ and a flow rate of $0.3 \mathrm{ml} / \mathrm{min}$. Mobile phases were $0.1 \% v / v$ formic acid in water and $0.1 \% v / v$ formic acid in acetonitrile, respectively. Mass spectrometry was run in ESIpositive mode, with probe capillary voltage set at $3,000 \mathrm{~V}$, a desolvation temperature of $350^{\circ} \mathrm{C}$, sheath gas of $11 \mathrm{ml} / \mathrm{h}$, and a nebulizer pressure of 50 psi (Chen et al., 2013).

\section{Expression and Analysis of luxI Homologous Genes in E. coli}

The luxI homologs, rhlI and lasI from YZ1 and P. aeruginosa ATCC27853 were PCR-amplified by using two pairs of specific primers (designed according to Accession No. CP006728.1). PCR products were ligated into $\mathrm{pBR} 322$ and transformed into $E$. coli O157:H7 strain 8624. The ahyI genes from YZ2 and $A$. hydrophila J-1 were transformed into E. coli F18 107/86 (primers were designed according to Accession No. X89469.1). Total RNA from recombinant $E$. coli strains were extracted using TRIzol as described previously (Yang et al., 2013). Data were normalized to the endogenous reference gene gapA and analyzed using the $2^{\Delta \Delta C T}$ method. For motility assays, E. coli strains were seeded in the middle of motility plates and motility halos were subsequently measured. For acid tolerance assays, E. coli strains were seeded in acidified LB ( $\mathrm{pH} 2.5$ ) supplemented with $1.0 \mathrm{mM}$ glutamate (Dyszel et al., 2010). Bacterial survival was enumerated from 0 to $2 \mathrm{~h}$.

\section{Statistics}

Quantitative data are shown as the mean \pm standard error of at least three independent experiments. Data were analyzed using two-tailed $t$-tests with asterisks indicating statistical significance $(p<0.05)$.

\section{RESULTS}

\section{Isolation of AHL-Producing Bacteria}

To screen cattle rumen fluid and pig intestinal samples for bacteria that produce AHLs, we filtered rumen fluids and scraped pig intestinal walls to collect samples, then inoculated LB plates 
with the filtered or PBS-washed contents. In each case, we screened $\sim 500$ isolated bacterial colonies for AHL production by seeding the individual colonies into LB for overnight growth and then extracted culture supernatants with ethyl acetate. These extracts were added to the AHL reporter strain JZA1 in the presence of X-gal to monitor AHLs in the extracted supernatants by quantifying AHL-dependent JZA1 $\beta$-galactosidase activity. We found that the culture supernatant derived from two single isolated colonies (here designated as YZ1 from cattle rumen fluid and YZ2 from pig intestinal contents) induced $\beta$-galactosidase activity (Figures 1A,B). $\beta$-galactosidase activity was highest when YZ1 was grown for at least $6 \mathrm{~h}$ (Figure 1A) and was more variable for YZ2 (Figure 1B).

To begin to characterize the isolated strains, the partial $16 \mathrm{~S}$ rDNA (1,528 bp) and AHL synthase genes (606 bp lasI/rhlI gene in YZ1 and 624 bp ahyI gene in YZ2) were sequenced. The sequencing data indicated strong homology (99\%) between Pseudomonas aeruginosa ATCC27853 and YZ1 (Figure 1C), and strong homology (99\%) between A. hydrophila J-1 and YZ2 (Figure 1D). Additionally, the colony odor and morphology of YZ1 and YZ2, oxidase production, and their ability to metabolize glucose, lactose, and sucrose were similar to that of other $P$. aeruginosa or A. hydrophila strains (data not shown). Hence, strain YZ1 was subsequently designated as P. aeruginosa YZ1, and YZ2 as A. hydrophila YZ2.

Because we initially used the JZA1 reporter assay to detect AHL-producing bacteria, we next sought to confirm these data using an independent method to avoid false positive results. We therefore utilized the biosensor strain C. violaceum CV026 in cross-streaking assays. Cross-streaking of YZ1 (Figure 1E) and YZ2 (Figure 1F), as well as the positive control strain Yersinia enterocolitica GIM1.266 on C. violaceum CV026 induced the formation of purple colonies, indicating that both $\mathrm{YZ1}$ and $\mathrm{YZ} 2$ secrete AHLs.

To differentiate between short- and long-side chain AHL productions, we next employed two different $E$. coli biosensor strains. Both E.coli/pSB401 and E. coli/pSB1142 contain the luxCDABE cassette. E.coli/pSB401 emits light in the presence of short chain AHLs, while E. coli/pSB1142 is activated by long chain AHLs. The addition of YZ1 extracts to $E$. coli/pSB1142 induced bioluminescence of this reporter strain, indicating that YZ1 synthesized long chain AHLs (Figure 2A). YZ1 extracts also activated E.coli/pSB401 bioluminescence to an extent greater than that of the negative control strain, E. coli DH5 $\alpha$ (Figure 2B). YZ2 extracts could only induce E.coli/pSB401 bioluminescence (Figures 2C,D). We therefore assumed that YZ1 secretes both short- and long-chain AHLs and YZ2 only secretes short-side chain AHLs.

\section{MS Analysis of YZ1/YZ2 AHLs}

To identify the AHLs produced by YZ1 and YZ2, we employed mass spectrometry. More than 30 types of AHL signals have been identified in various bacterial QS systems (Gould et al., 2006). Previous papers have summarized the specific $\mathrm{m} / \mathrm{z}$ data of ions from each AHL, which provided us sufficient information with which to identify the specific AHL produced by $\mathrm{YZ1}$
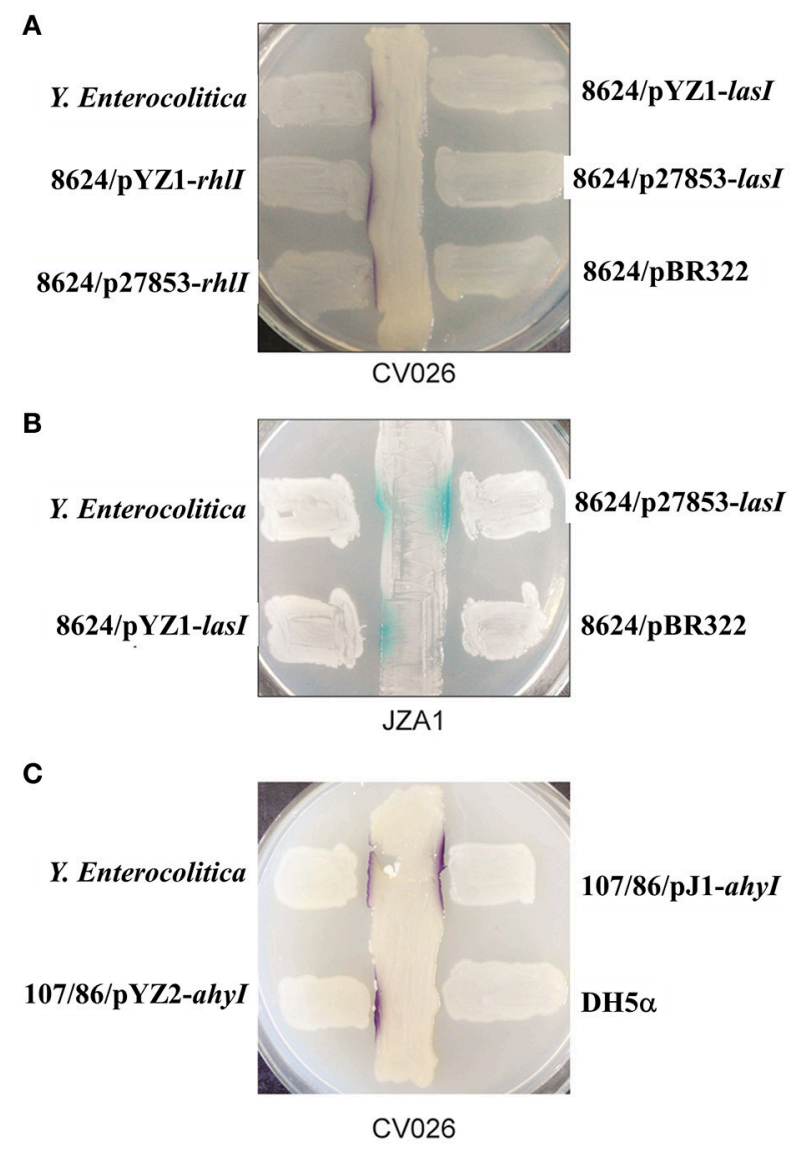

FIGURE 4 | Cross-streaking assays for Al-1 activity from E. coli 8624 transformed with lasl or rhll and E. coli 107/86 transformed with ahyl. (A) Biosensor strain C. violaceum CV026-based cross-streaking assay. The indicated E. coli 8624 recombinant strains were struck across the CV026 biosensor strain to detect short-chain AHLs (purple color). (B) Biosensor strain JZA1 assay. The indicated $E$. coli 8624 recombinant strains were struck across the JZA1 biosensor strain to detect long-chain AHLs (blue color). (C) CV026-based cross-streaking assay. The indicated E. coli 107/86 recombinant strain was struck across the CV026 biosensor strain to detect short-chain AHLs.

and YZ2 (Ortori et al., 2007). By analyzing the contents of concentrated supernatants, in YZ1 we detected very similar mass peaks (Figures 3A-C) compared with C4-AHL (m/z 172.60), C8AHL (m/z 228.07), and 3-oxo-C12-AHL (m/z 298.72), and in YZ2 we detected similar mass peaks (Figure 3D) compared with C4-AHL(m/z 172.40).

\section{Expression of luxl Homologous Genes in E. coli}

$P$. aeruginosa possesses two AHL-dependent QS circuits, rhlRI and lasRI systems, which are responsible for the production of short- and long-chain AHLs, respectively (Chen et al., 2013). The luxI homologs rhlI and lasI from both $P$. aeruginosa YZ1 and ATCC27853 were PCR-amplified and cloned into pBR322. The recombinant plasmids were transformed into E. coli O157:H7 strain 8624 , to determine whether endogenous expression of 


\section{A}

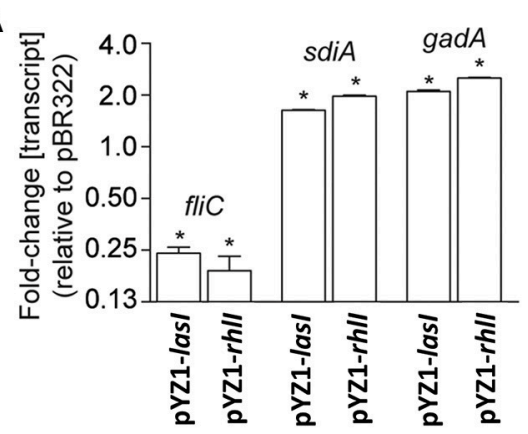

B

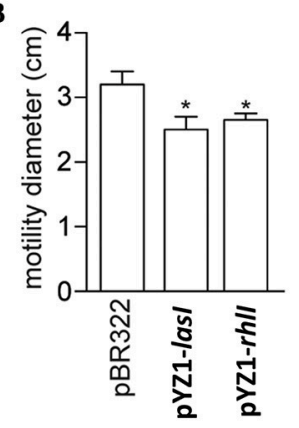

C

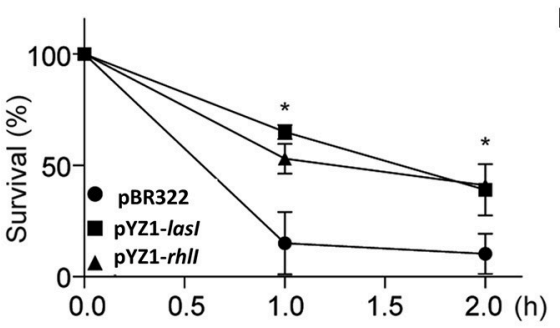

D

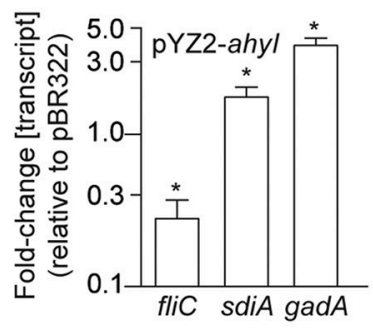

E

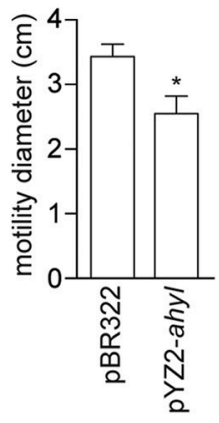

F

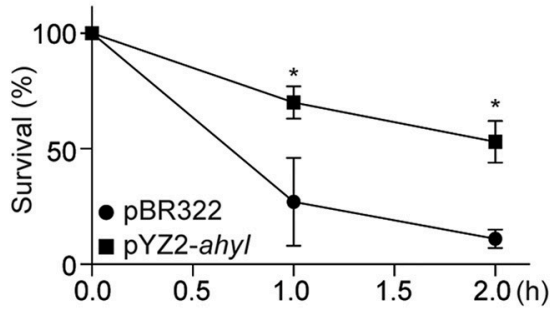

FIGURE 5 | Phenotypic analysis of E. coli 8624 and 107/86 recombinant strains. (A) Relative fliC, sdiA, and gadA transcript abundance (as compared with E. coli 8624 transformed with pBR322) are plotted as a function of $E$. coli transformation with luxl homologs. Asterisks indicate significantly different gene expression, as compared with E. coli 8624 transformed with pBR322. (B) Motility diameters were measured after $12 \mathrm{~h}$ growth of the indicated strains on $0.3 \%$ swim agar plates. Asterisks indicate significantly different motility halos, as compared with E. coli 8624 transformed with pBR322. (C) Acid tolerance was quantified by enumerating bacteria survival as a function of time after growth seeded in acidified LB (pH 2.5) supplemented with 1.0 mM glutamate. Asterisks indicate significantly different survival, as compared with E. coli 8624 transformed with pBR322. (D) Relative fliC, sdiA, and gadA transcript abundance (as compared with E. coli 107/86 transformed with pBR322) are plotted as a function of E. coli transformation with ahyl from YZ2. Asterisks indicate significantly different gene expression, as compared with E. coli 107/86 transformed with pBR322. (E) Motility diameters were measured after $12 \mathrm{~h}$ growth of the indicated strains on $0.3 \%$ swim agar plates. Asterisks indicate significantly different motility halos, as compared with E. coli 107/86 transformed with pBR322. (F) Acid tolerance was quantified by enumerating bacteria survival as a function of time after growth seeded in acidified LB ( $\mathrm{pH}$ 2.5) supplemented with $1.0 \mathrm{mM}$ glutamate. Asterisks indicate significantly different survival, as compared with E. coli 107/86 transformed with pBR322.

these genes would confer upon E. coli the ability to express AHLs. The same method was also used to study the ahyI gene from A. hydrophila, which was transformed into F18 E. coli $107 / 86$.

Cross-streaking of E. coli 8624 expressing rhlI from both P. aeruginosa YZ1 and ATCC27853 on C. violaceum CV026 induced the formation of purple colonies, suggesting that expressing rhlI in E. coli conferred the expression of short chain AHLs (Figure 4A; Pearson et al., 1995). We subsequently used the JZA1 reporter assay to quantify long-chain AHLdependent $\beta$-galactosidase activity. These cross-streaking experiments also induced $\beta$-galactosidase activity, suggesting long chain AHL production in E. coli after transformation with lasI (Figure 4B). Cross-streaking of E. coli107/86 expressing ahyI from both $A$. hydrophila J-1 and YZ2 on CV026 induced the formation of purple colonies, suggesting that expressing ahyI conferred the expression of short chain AHLs (Figure 4C).

Expressing the luxI homologs lasI and rhlI in E. coli affected the expression of several genes known to be regulated by AHLs, including $f l i C$, $g a d A$, and $s d i A$ (Figure 5A). Consistent with these gene expression data, E. coli motility was inhibited (Figure 5B), whereas acid tolerance was enhanced (Figure 5C). Similar to the data obtained using YZ1, we also found that expressing ahyI from YZ2 in E. coli affected E. coli virulence gene expression, motility, and acid resistance (Figures 5D-F). 


\section{DISCUSSION}

The identification of QS signaling molecules and the bacterial strains that produce these molecules is of considerable significance (Sperandio, 2010; Soares and Ahmer, 2011). A variety of bioassay strains have been developed to facilitate the detection of AHLs. Ideally, such a strain would contain an easily assayable reporter construct, lack endogenous AHL synthases, and be able to respond to exogenous AHLs (Zhu et al., 2003). However, most bioassay strains detect only a subset of AHLs. A. tumefaciens JZA1 is considered to be perhaps the most sensitive bioassay strain and can detect a wide range of AHLs (Zhu et al., 2003). However, it had not, to our knowledge, previously been utilized successfully to screen AHL-producing bacteria in cattle rumen fluids or pig intestinal contents (Erickson et al., 2002; Smith et al., 2008).

We therefore introduced enrichment, extraction, and evaporation steps to concentrate culture supernatants derived from rumen fluids or intestinal contents. By employing these modifications, we identified the AHL-producing bacterium $P$. aeruginosa $\mathrm{YZ1}$ in cattle rumen, and $A$. hydrophila $\mathrm{YZ2}$ in pig intestine. A. hydrophila was previously characterized for its quorum sensing functions upon Salmonella enterica in the gastrointestinal tract of turtles (Smith et al., 2008). Mass spectrometry analysis indicated the production of AHLs by YZ1 and YZ2. By cloning luxI homologs from YZ1 or YZ2, we conferred upon E. coli the ability to synthesize AHLs, to examine whether AHLs from specific environment could induce QS related pathogenic gene in E. coli.

E. coli inhabit the intestinal environment, and play important roles in humans and many other animals. Because its lack in AHL production, the simplest hypothesis is that in intestinal environment $E$. coli use SdiA to detect exogenous AHL, through which its pathogenicity could be regulated by these AHL-positive strains. However, this hypothesis appears to be incorrect, as chemical extractions and a Salmonella SdiA reporter both failed to detect AHLs within mammalian intestines (Soares and Ahmer, 2011; Swearingen et al., 2012). The failure of screening for AHL-positive bacteria in the digestive tract has directly affected the in-depth study for the synergistic regulation mechanism in pathogenicity of the intestinal bacteria, especially E. coli. Although this paper failed to answer whether YZ1 and YZ2 could synthesize AHL in the gut environment or synthesize sufficient

\section{REFERENCES}

Ahmer, B. M. (2004). Cell-to-cell signalling in Escherichia coli and Salmonella enterica. Mol. Microbiol. 52, 933-945. doi: 10.1111/j.1365-2958.2004.04054.x

Andrade, G. I., Coura, F. M., Santos, E. L., Ferreira, M. G., Galinari, G. C., Facury, F. E., et al. (2012). Identification of virulence factors by multiplex PCR in Escherichia coli isolated from calves in Minas Gerais, Brazil. Trop. Anim. Health Prod. 44, 1783-1790. doi: 10.1007/s11250-012-0139-8

Barlow, R. S., and Mellor, G. E. (2010). Prevalence of enterohemorrhagic Escherichia coli serotypes in Australian beef cattle. Foodborne Pathog. Dis. 7, 1239-1245. doi: 10.1089/fpd.2010.0574

Bertschinger, H. U., Bachmann, M., Mettler, C., Pospischil, A., Schraner, E. M., Stamm, M., et al. (1990). Adhesive fimbriae produced in vivo by Escherichia
AHL to activate QS-1 system in E. coli, it still emphasize the possibility that $E$. coli in relative environments could be regulated by AHL signals from other AHL-positive strains.

Biosensors have detected acyl-HSLs in chemical extracts of cow rumens, and rumen AHLs are known to repress expression of the E. coli LEE and activate gadA expression to improve acid tolerance (Dyszel et al., 2010; Sperandio, 2010; Sheng et al., 2013), while pig intestinal AHLs still have unclear functions in regulating E. coli virulence. Although not all AHL signals in nature environment could bind to E. coli SdiA and regulate its pathogenicity, function of QS-1 signals from $P$. aeruginosa YZ1 and A. hydrophila YZ2 upon E. coli virulence have been confirmed, which strongly support that hypothesis. The data described here represent a step forward in the successful isolation and characterization of AHL-synthase positive bacteria from cattle rumen and pig intestines, and offer us a new vision to consider the multi-relationship between E. coli and gastrointestinal tract in infection process through quorum sensing.

\section{AUTHOR CONTRIBUTIONS}

GZ, PH, and YY conceived and designed the experiments. MZ isolated and analyzed YZ1. YY isolated and analyzed YZ2. YY and $\mathrm{HC}$ wrote this paper.

\section{ACKNOWLEDGMENTS}

Professor Yongjie Liu (Nanjing Agricultural University, China) provided CV026. Professor Jun Zhu (University of Pennsylvania, USA) provided JZA1. Professor Miguel Camara (University of Nottingham, United Kingdom) provided $E$. coli pSB401 and pSB1142. This study was supported by grants from Natural Science Foundation of Jiangsu Province (BK20150442), 13th Five-Year National Key Development Program(2016YFD0501000, 2016YFD0500905), the Chinese National Science Foundation Grants (Nos. 31502075, 31672579,31270171, 31072136, 30771603), the Genetically Modified Organisms Technology Major Project of China (2014ZX08006-001B), Program for ChangJiang Scholars and Innovative Research Team In University PCSIRT (IRT0978), 948 programme from Ministry of Agriculture of the People's Republic of China (grant No. 2011-G24), the Priority Academic Program Development of Jiangsu Higher Education Institutions.

coli $\mathrm{O} 139: \mathrm{K} 12(\mathrm{~B}): \mathrm{H} 1$ associated with enterotoxaemia in pigs. Vet. Microbiol. 25, 267-281.

Borriello, G., Lucibelli, M. G., De Carlo, E., Auriemma, C., Cozza, D., Ascione, G., et al. (2012). Characterization of enterotoxigenic E. coli (ETEC), Shiga-toxin producing E. coli (STEC) and necrotoxigenic E. coli (NTEC) isolated from diarrhoeic Mediterranean water buffalo calves (Bubalus bubalis). Res. Vet. Sci. 93, 18-22. doi: 10.1016/j.rvsc.2011. 05.009

Boyen, F., Eeckhaut, V., Van Immerseel, F., Pasmans, F., Ducatelle, R., and Haesebrouck, F. (2009). Quorum sensing in veterinary pathogens: mechanisms, clinical importance and future perspectives. Vet. Microbiol. 135, 187-195. doi: 10.1016/j.vetmic.2008. 12.025 
Chen, J. W., Chin, S., Tee, K. K., Yin, W. F., Choo, Y. M., and Chan, K. G. (2013). $\mathrm{N}$-acyl homoserine lactone-producing Pseudomonas putida strain T2-2 from human tongue surface. Sensors 13, 13192-13203. doi: 10.3390/s131013192

da Silva, S. A., Valadares, G. F., Penatti, M. P., Brito, B. G., and da Silva Leite.D (2001). Escherichia coli strains from edema disease: O serogroups, and genes for Shiga toxin, enterotoxins, and F18 fimbriae. Vet. Microbiol. 80, 227-233. doi: 10.1016/S0378-1135(01)00316-9

D'Amato, R. F., Thornsberry, C., Baker, C. N., and Kirven, L. A. (1975). Effect of calcium and magnesium ions on the susceptibility of Pseudomonas species to tetracycline, gentamicin polymyxin B, and carbenicillin. Antimicrob. Agents Chemother. 7, 596-600. doi: 10.1128/AAC.7.5.596

Duan, Q., Zhou, M., Zhu, X., Bao, W., Wu, S., Ruan, X., et al. (2012). The flagella of F18ab Escherichia coli is a virulence factor that contributes to infection in a IPEC-J2 cell model in vitro. Vet. Microbiol. 160, 132-140. doi: 10.1016/j.vetmic.2012.05.015

Duan, Q., Zhou, M., Zhu, X., Yang, Y., Zhu, J., Bao, W., et al. (2013). Flagella from F18+Escherichia coli play a role in adhesion to pig epithelial cell lines. Microb. Pathog. 55, 32-38. doi: 10.1016/j.micpath.2012.09.010

Dyszel, J. L., Soares, J. A., Swearingen, M. C., Lindsay, A., Smith, J. N., and Ahmer, B. M. (2010). E. coli K-12 and EHEC genes regulated by SdiA. PLoS ONE 5:e8946. doi: 10.1371/journal.pone.0008946

Erickson, D. L., Nsereko, V. L., Morgavi, D. P., Selinger, L. B., Rode, L. M., and Beauchemin, K. A. (2002). Evidence of quorum sensing in the rumen ecosystem: detection of $\mathrm{N}$-acyl homoserine lactone autoinducers in ruminal contents. Can. J. Microbiol. 48, 374-378. doi: 10.1139/w02-022

Frydendahl, K. (2002). Prevalence of serogroups and virulence genes in Escherichia coli associated with postweaning diarrhoea and edema disease in pigs and a comparison of diagnostic approaches. Vet. Microbiol. 85, 169-182. doi: 10.1016/S0378-1135(01)00504-1

Fuqua, C., and Winans, S. C. (1996). Conserved cis-acting promoter elements are required for density-dependent transcription of Agrobacterium tumefaciens conjugal transfer genes. J. Bacteriol. 178, 435-440. doi: $10.1128 /$ jb.178.2.435-440.1996

Gould, T. A., Herman, J., Krank, J., Murphy, R. C., and Churchill, M. E. (2006). Specificity of acyl-homoserine lactone synthases examined by mass spectrometry. J. Bacteriol. 188, 773-783. doi: 10.1128/JB.188.2.773-783.2006

Havens, P. L., Dunne, W. M., and Burd, E. M. (1992). Effects of human intravenous immune globulin on diarrhea caused by Shiga-like toxin I and Shiga-like toxin II in infant rabbits. Microbiol. Immunol. 36, 1077-1085. doi: 10.1111/j.1348-0421.1992.tb02111.x

Hughes, D. T., Terekhova, D. A., Liou, L., Hovde, C. J., Sahl, J. W., Patankar, A. V., et al. (2010). Chemical sensing in mammalian hostbacterial commensal associations. Proc. Natl. Acad. Sci. U.S.A. 107, 9831-9836. doi: $10.1073 /$ pnas. 1002551107

Kanamaru, K., Kanamaru, K., Tatsuno, I., Tobe, T., and Sasakawa, C. (2000). SdiA, an Escherichia coli homologue of quorum-sensing regulators, controls the expression of virulence factors in enterohaemorrhagic Escherichia coli O157:H7. Mol. Microbiol. 38, 805-816. doi: 10.1046/j.1365-2958.2000.02171.x

Latifi, A., Winson, M. K., Foglino, M., Bycroft, B. W., Stewart, G. S., Lazdunski, A., et al. (1995). Multiple homologues of LuxR and LuxI control expression of virulence determinants and secondary metabolites through quorum sensing in Pseudomonas aeruginosa PAO1. Mol. Microbiol. 17, 333-343. doi: 10.1111/j.1365-2958.1995.mmi_17020333.x

Lu, Y., Zeng, J., Wu, B., E, S., Wang, L., Cai, R., et al. (2017). Quorum sensing $\mathrm{N}$-acyl homoserine lactones-SdiA suppresses Escherichia coli-Pseudomonas aeruginosa conjugation through inhibiting traI expression. Front. Cell. Infect. Microbiol. 7:7. doi: 10.3389/fcimb.2017.00007

Mainil, J. G., Jacquemin, E., Pohl, P., Kaeckenbeeck, A., and Benz, I. (2002). DNA sequences coding for the F18 fimbriae and AIDA adhesin are localised on the same plasmid in Escherichia coli isolates from piglets. Vet. Microbiol. 86, 303-311. doi: 10.1016/S0378-1135(02)00019-6

Nguyen, Y., Nguyen, N. X., Rogers, J. L., Liao, J., MacMillan, J. B., Jiang, Y., et al. (2015). Structural and mechanistic roles of novel chemical ligands on the SdiA quorum-sensing transcription regulator. MBio 6:e2429-14. doi: $10.1128 / \mathrm{mBio} .02429-14$

Ortori, C. A., Atkinson, S., Chhabra, S. R., Cámara, M., Williams, P., and Barrett, D. A. (2007). Comprehensive profiling of $\mathrm{N}$-acylhomoserine lactones produced by Yersinia pseudotuberculosis using liquid chromatography coupled to hybrid quadrupole-linear ion trap mass spectrometry. Anal. Bioanal. Chem. 387, 497-511. doi: 10.1007/s00216-006-0710-0

Palaniyandi, S., Mitra, A., Herren, C. D., Zhu, X., and Mukhopadhyay, S. (2013). LuxS contributes to virulence in avian pathogenic Escherichia coli O78:K80:H9. Vet. Microbiol. 166, 567-575. doi: 10.1016/j.vetmic.2013.07.009

Pearson, J. P., Passador, L., Iglewski, B. H., and Greenberg, E. P. (1995). A second $\mathrm{N}$-acylhomoserine lactone signal produced by Pseudomonas aeruginosa. Proc. Natl. Acad. Sci. U.S.A. 92, 1490-1494. doi: 10.1073/pnas.92.5.1490

Rahmati, S., Yang, S., Davidson, A. L., and Zechiedrich, E. L. (2002). Control of the AcrAB multidrug efflux pump by quorum-sensing regulator SdiA. Mol. Microbiol. 43, 677-685. doi: 10.1046/j.1365-2958.2002.02773.x

Reading, N. C., and Sperandio, V. (2006). Quorum sensing: the many languages of bacteria. FEMS Microbiol. Lett. 254, 1-11. doi: 10.1111/j.1574-6968.2005.00001.x

Sheng, H., Nguyen, Y. N., Hovde, C. J., and Sperandio, V. (2013). SdiA aids enterohemorrhagic Escherichia coli carriage by cattle fed a forage or grain diet. Infect. Immun. 81, 3472-3478. doi: 10.1128/IAI.00702-13

Shimada, T., Shimada, K., Matsui, M., Kitai, Y., Igarashi, J., Suga, H., et al. (2014). Roles of cell division control factor SdiA: recognition of quorum sensing signals and modulation of transcription regulation targets. Genes Cells 19, 405-418. doi: $10.1111 /$ gtc. 12139

Smith, J. L., Fratamico, P. M., and Yan, X. (2011). Eavesdropping by bacteria: the role of SdiA in Escherichia coli and Salmonella enterica serovar Typhimurium quorum sensing. Foodborne Pathog. Dis. 8, 169-178. doi: 10.1089/fpd.2010.0651

Smith, J. N., Dyszel, J. L., Soares, J. A., Ellermeier, C. D., Altier, C., Lawhon, S. D., et al. (2008). SdiA, an $\mathrm{N}$-acylhomoserine lactone receptor, becomes active during the transit of Salmonella enterica through the gastrointestinal tract of turtles. PLoS ONE 3:e2826. doi: 10.1371/journal.pone.0002826

Soares, J. A., and Ahmer, B. M. (2011). Detection of acyl-homoserine lactones by Escherichia and Salmonella. Curr. Opin. Microbiol. 14, 188-193. doi: 10.1016/j.mib.2011.01.006

Sperandio, V. (2010). SdiA sensing of acyl-homoserine lactones by enterohemorrhagic E. coli (EHEC) serotype O157:H7 in the bovine rumen. Gut Microbes 1, 432-435. doi: 10.4161/gmic.1.6.14177

Sperandio, V., Torres, A. G., and Kaper, J. B. (2002). Quorum sensing Escherichia coli regulators $\mathrm{B}$ and $\mathrm{C}(\mathrm{Q} s \mathrm{BC})$ : a novel two-component regulatory system involved in the regulation of flagella and motility by quorum sensing in E. coli. Mol. Microbiol. 43, 809-821. doi: 10.1046/j.1365-2958.2002.02803.x

Swearingen, M. C., Sabag-Daigle, A., and Ahmer, B. M. M. (2012). Are there acylhomoserine lactones within mammalian intestines? J. Bacteriol. 195, 173-179. doi: 10.1128/JB.01341-12

Tran, P. T., Simon, J. A., and Liskay, R. M. (2001). Interactions of Exolp with components of MutLalpha in Saccharomyces cerevisiae. Proc. Natl. Acad. Sci. U.S.A. 98, 9760-9765. doi: 10.1073/pnas.161175998

Van Houdt, R., Aertsen, A., Moons, P., Vanoirbeek, K., and Michiels, C. W. (2006). N-acyl-L-homoserine lactone signal interception by Escherichia coli. FEMS Microbiol. Lett. 256, 83-89. doi: 10.1111/j.1574-6968.2006. 00103.x

Walters, M., and Sperandio, V. (2006). Quorum sensing in Escherichia coli and Salmonella. Int. J. Med. Microbiol. 296, 125-131. doi: 10.1016/j.ijmm.2006.01.041

Waters, C. M., Lu, W., Rabinowitz, J. D., and Bassler, B. L. (2008). Quorum sensing controls biofilm formation in Vibrio cholerae through modulation of cyclic di-GMP levels and repression of vpsT. J. Bacteriol. 190, 2527-2536. doi: 10.1128/JB.01756-07

Wei, Y., Lee, J. M., Smulski, D. R., and LaRossa, R. A. (2001). Global impact of sdiA amplification revealed by comprehensive gene expression profiling of Escherichia coli. J. Bacteriol. 183, 2265-2272. doi: 10.1128/JB.183.7.2265-2272.2001

Winson, M. K., Swift, S., Fish, L., Throup, J. P., Jørgensen, F., Chhabra, S. R., et al. (1998). Construction and analysis of luxCDABE-based plasmid sensors for investigating $\mathrm{N}$-acyl homoserine lactone-mediated quorum sensing. FEMS Microbiol. Lett. 163, 185-192. doi: 10.1111/j.1574-6968.1998. tb13044.x

Yakhnin, H., Baker, C. S., Berezin, I., Evangelista, M. A., Rassin, A., Romeo, T., et al. (2011). CsrA represses translation of sdiA, which encodes the Nacylhomoserine-L-lactone receptor of Escherichia coli, by binding exclusively 
within the coding region of sdiA mRNA. J. Bacteriol. 193, 6162-6170. doi: 10.1128/JB.05975-11

Yamamoto, K., Yata, K., Fujita, N., and Ishihama, A. (2001). Novel mode of transcription regulation by SdiA, an Escherichia coli homologue of the quorum-sensing regulator. Mol. Microbiol. 41, 1187-1198. doi: 10.1046/j.1365-2958.2001.02585.x

Yan, Y., Chen, H., and Lu, C. (1996). Purification and characterization of S-layer protein from Aeromonas hydrophila. Wei Sheng Wu Xue Bao 36, 144-150.

Yang, Y., Yao, F., Zhou, M., Zhu, J., Zhang, X., Bao, W., et al. (2013). F18ab Escherichia coli flagella expression is regulated by acyl-homoserine lactone and contributes to bacterial virulence. Vet. Microbiol. 165, 378-383. doi: 10.1016/j.vetmic.2013.04.020

Yang, Y., Zhou, M., Hou, H., Zhu, J., Yao, F., Zhang, X., et al. (2014). Quorum-sensing gene luxS regulates flagella expression and Shiga-like toxin production in F18ab Escherichia coli. Can. J. Microbiol. 60, 355-361. doi: 10.1139/cjm-2014-0178

Yao, Y., Martinez-Yamout, M. A., Dickerson, T. J., Brogan, A. P., Wright, P. E., and Dyson, H. J. (2006). Structure of the Escherichia coli quorum sensing protein SdiA: activation of the folding switch by acyl homoserine lactones. J. Mol. Biol. 355, 262-273. doi: 10.1016/j.jmb.2005.10.041

Yin, W. F., Purmal, K., Chin, S., Chan, X. Y., Koh, C. L., and Sam, C. K., et al. (2012). N-acyl homoserine lactone production by Klebsiella pneumoniae isolated from human tongue surface. Sensors 12, 3472-3483. doi: 10.3390/s120 303472
Zhang, Y., Ma, Q., Su, B., Chen, R., Lin, J., Lin, Z., et al. (2018). A study on the role that quorum sensing play in antibiotic-resistant plasmid conjugative transfer in Escherichia coli. Ecotoxicology 27, 209-216. doi: 10.1007/s10646-017-1886-0

Zhu, J., Chai, Y., Zhong, Z., Li, S., and Winans, S. C. (2003). Agrobacterium bioassay strain for ultrasensitive detection of $\mathrm{N}$-acylhomoserine lactone-type quorum-sensing molecules: detection of autoinducers in Mesorhizobium huakuii. Appl. Environ. Microbiol. 69, 6949-6953. doi: 10.1128/AEM.69.11.6949-6953.2003

Zhu, J., Yin, X., Yu, H., Zhao, L., Sabour, P., and Gong, J. (2011). Involvement of quorum sensing and heat-stable enterotoxin a in cell damage caused by a porcine enterotoxigenic Escherichia coli strain. Infect. Immun. 79, 1688-1695. doi: 10.1128/IAI.01281-10

Conflict of Interest Statement: The authors declare that the research was conducted in the absence of any commercial or financial relationships that could be construed as a potential conflict of interest.

Copyright $\odot 2018$ Yang, Zhou, Hardwidge, Cui and Zhu. This is an open-access article distributed under the terms of the Creative Commons Attribution License (CC $B Y)$. The use, distribution or reproduction in other forums is permitted, provided the original author(s) and the copyright owner are credited and that the original publication in this journal is cited, in accordance with accepted academic practice. No use, distribution or reproduction is permitted which does not comply with these terms. 